\title{
IMAGING DIAGNOSIS OF A SHOULDER TUMOR - CASE PRESENTATION
}

doi: $10.2478 /$ rojost-2018-0050

\author{
R. Turcu ${ }^{1,2}$, A. Barbilian ${ }^{1,2}$ \\ "Dr. Carol Davila" Universitary Emergency Central Military Hospital, Bucharest, Romania \\ The Orthopaedics - Traumathology Clinic \\ 2 "Carol Davila" University of Medicine and Pharmacy, Bucharest, Romania
}

Introduction. The most common and used molecular imaging techniques used in tumor pathology are the following: optics such as fluoroscopy, bioluminescence, and spectroscopy, radionuclides such as positron emission computed tomography, magnetic resonance with or without contrast substance, ultrasound and computed tomography.

Material and method. A 39-year-old male patient accused having a shoulder tumor beginning 6 months before with a slow increase in volume without symptoms, pain, local temperature changes, and neurological phenomena. The clinical examination revealed the presence of an elastic consistency tumor, adherent to the bone but movable in soft adjacent tissues, without spontaneous and palpation pain, and local swelling. There was a slight functional embarrassment in conducting the abduction maneuver, but without limiting the amplitude of the movement.

Results. Radiography of the shoulder did not reveal any significant changes. Magnetic resonance imaging of the shoulder and contrast-enhanced magnetic resonance imaging of the shoulder were performed. The evoked changes required a computed tomography of the shoulder.

Conclusion. The thorough analysis of the imaging investigations and the clinical, paraclinical, and biological context of the patient will lead to the indication of the treatment and the optimal surgical time.

Keywords: shoulder tumor, contrast-enhanced magnetic resonance imaging, computed tomography 